\title{
Existence and Stability Results for Impulsive Fractional $q$-Difference Equation
}

\author{
Min Jiang1*, Rengang Huang2 \\ ${ }^{1}$ College of Computer and Information Engineering, Guizhou Minzu University, Guiyang, China \\ ${ }^{2}$ College of Business, Guizhou Minzu University, Guiyang, China \\ Email: *minjiang0701@163.com
}

How to cite this paper: Jiang, $M$. and Huang, R.G. (2020) Existence and Stability Results for Impulsive Fractional $q$-Difference Equation. Journal of Applied Mathematics and Physics, 8, 1413-1423.

https://doi.org/10.4236/jamp.2020.87107

Received: June 21, 2020

Accepted: July 27, 2020

Published: July 30, 2020

Copyright (C) 2020 by author(s) and Scientific Research Publishing Inc. This work is licensed under the Creative Commons Attribution International License (CC BY 4.0).

http://creativecommons.org/licenses/by/4.0/

(c) (i) Open Access

\begin{abstract}
In this paper, we study the boundary value problem for an impulsive fractional $q$-difference equation. Based on Banach's contraction mapping principle, the existence and Hyers-Ulam stability of solutions for the equation which we considered are obtained. At last, an illustrative example is given for the main result.
\end{abstract}

\section{Keywords}

Impulsive Fractional $q$-Difference Equation, Hyers-Ulam Stability, Existence, $q$-Calculus

\section{Introduction}

The $q$-calculus or quantum calculus is an old subject that was initially developed by Jackson [1]; basic definitions and properties of $q$-calculus can be found in [2]. The fractional $q$-calculus had its origin in the works by Al-Salam [3] and Agarwal [4]. But the definitions mentioned above about $q$-calculus can't be applied to impulse points $t_{k}, k \in \mathbb{Z}$, such that $t_{k} \in(q t, t)$. In [5], the authors defined the concepts of fractional $q$-calculus by defining a $q$-shifting operator ${ }_{a} \Phi_{q}(m)=q m+(1-q) a, m, a \in \mathbb{R}$. Using the $q$-shifting operator, the fractional impulsive $q$-difference equation was defined. In paper [5] [6] [7], the authors discussed the existence of solutions for the fractional impulsive $q$-difference equation with Riemann-Liouville and Caputo fractional derivatives respectively. Some other results about $q$-difference equations can be found in papers [8]-[16] and the references cited therein. Dumitru Baleanu et al. discussed the stability of non-autonomous systems with the $q$-Caputo fractional derivatives in reference [17]. However, the existence and stability of solutions for the fractional impul- 
sive $q$-difference have not been yet studied.

Motivated greatly by the above mentioned excellent works, in this paper we investigate the following fractional impulsive $q$-difference equation with $q$-integral boundary conditions:

$$
\left\{\begin{array}{l}
{ }_{t_{k}}^{c} D_{q_{k}}^{\alpha_{k}} x(t)=f(t, x(t)), t \in J_{k} \subseteq J=[0, T], t \neq t_{k}, \\
\Delta x\left(t_{k}\right)=x\left(t_{k}^{+}\right)-x\left(t_{k}\right)=\varphi_{k}\left(x\left(t_{k}\right)\right), k=1,2, \cdots, m, \\
\eta_{1} x(0)+\eta_{2} x(T)=\mu \sum_{k=0}^{m}{ }_{t_{k}} I_{q_{k}}^{\beta_{k}} x\left(t_{k+1}\right) .
\end{array}\right.
$$

where ${ }_{t_{k}}^{c} D_{q_{k}}^{\alpha_{k}}$ is the fractional $q_{k}$-derivative of the Caputo type of order $\alpha_{k}$ on $J_{k}, 0<\alpha_{k}<1,0<q_{k}<1, \quad J_{0}=\left[0, t_{1}\right], \quad J_{0}=\left[0, t_{1}\right], k=1,2, \cdots, m$, $\varphi_{k} \in C(\mathbb{R}, \mathbb{R}), \quad f \in C(J \times \mathbb{R}, \mathbb{R}) . \quad{ }_{t_{k}} I_{q_{k}}^{\beta_{k}}$ denotes the Riemann-Liouville $q_{k}$ fractional integral of order $\beta_{k}>0$ on $J_{k}, k=0,1,2, \cdots, m$ and $\eta_{1}, \eta_{2}, \mu$ are three constants.

\section{Preliminaries on $q$-Calculus and Lemmas}

Here we recall some definitions and fundamental results on fractional $q$-integral and fractional $q$-derivative, for the full theory for which one is referred to [5] [6] [7].

For $q \in(0,1)$, we define a $q$-shifting operator as ${ }_{a} \Phi_{q}(m)=q m+(1-q) a$. The new power of $q$-shifting operator is defined as ${ }_{a}(n-m)_{q}^{(0)}=1$,

$$
\begin{aligned}
{ }_{a}(n-m)_{q}^{(k)} & =\prod_{i=0}^{k-1}\left(n-{ }_{a} \Phi_{q}^{i}(m)\right), \quad k \in \mathbb{N} \bigcup\{0\}, n \in \mathbb{R} . \text { If } v \in \mathbb{R}, \text { then } \\
{ }_{a}(n-m)_{q}^{(v)} & =n^{v} \prod_{i=0}^{\infty} \frac{1-\frac{a}{n} \Phi_{q}^{i}\left(\frac{m}{n}\right)}{1-\frac{a}{n} \Phi_{q}^{i+v}\left(\frac{m}{n}\right)} .
\end{aligned}
$$

The $q$-derivative of a function $f$ on interval $[a, b]$ is defined by

$$
\left({ }_{a} D_{q} f\right)(t)=\frac{f(t)-f\left({ }_{a} \Phi_{q}(t)\right)}{(1-q)(t-a)}, t \neq a,\left({ }_{a} D_{q} f\right)(a)=\lim _{t \rightarrow a}\left({ }_{a} D_{q} f\right)(t) .
$$

The $q$-integral of a function $f$ defined on the interval $[a, b]$ is given by

$$
\left({ }_{a} I_{q} f\right)(t)=\int_{a}^{t} f(s)_{a} \mathrm{~d} s=(1-q)(t-a) \sum_{i=0}^{\infty} q^{i} f\left({ }_{a} \Phi_{q^{i}}(t)\right), t \in[a, b] .
$$

Some results about operator ${ }_{a} D_{q}$ and ${ }_{a} I_{q}$ can be found in references [5]. Let us define fractional $q$-derivative and $q$-integral on interval $[a, b]$ and outline some of their properties [5] [6] [7].

Definition 1 [5] The fractional $q$-derivative of Riemann-Liouville type of order $v \geq 0$ on interval $[a, b]$ is defined by $\left({ }_{a} D_{q}^{0} f\right)(t)=f(t)$ and

$$
\left({ }_{a} D_{q}^{v} f\right)(t)=\left({ }_{a} D_{q a}^{l} I_{q}^{I-v} f\right)(t), v>0,
$$

where $l$ is the smallest integer greater than or equal to $v$.

Definition 2 [5] Let $\alpha \geq 0$ and $f$ be a function defined on $[a, b]$. The 
fractional $q$-integral of Riemann-Liouville type is given by $\left({ }_{a} I_{q}^{0} f\right)(t)=f(t)$ and

$$
\left({ }_{a} I_{q}^{\alpha} f\right)(t)=\frac{1}{\Gamma_{q}(\alpha)} \int_{a}^{t}\left(t-{ }_{a} \Phi_{q}(s)\right)_{q}^{\alpha-1} f(s)_{a} \mathrm{~d}_{q} s, \alpha>0, t \in[a, b] .
$$

Lemma 1 [5] Let $\alpha, \beta \in \mathbb{R}^{+}$and $f$ be a continuous function on $[a, b], a \geq 0$. The Riemann-Liouville fractional $q$-integral has the following semi-group property

$$
{ }_{a} I_{q}^{\beta} I_{q}^{\alpha} I^{\alpha} f(t)={ }_{a} I_{q}^{\alpha}{ }_{a} I_{q}^{\beta} f(t)={ }_{a} I_{q}^{\alpha+\beta} f(t) .
$$

Lemma 2 [5] Let $f$ be a $q$-integrable function on $[a, b]$. Then the following equality holds

$$
{ }_{a} D_{q a}^{\alpha} I_{q}^{\alpha} f(t)=f(t), \text { for } \alpha>0, t \in[a, b] .
$$

Lemma 3 [5] Let $\alpha>0$ and $p$ be a positive integer. Then for $t \in[a, b]$ the following equality holds

$$
{ }_{a} I_{q a}^{\alpha} D_{q}^{p} f(t)={ }_{a} D_{q}^{p} I_{q} I_{q}^{\alpha} f(t)-\sum_{k=0}^{p-1} \frac{(t-a)^{\alpha-p+k}}{\Gamma_{q}(\alpha+k-p+1)}{ }_{a} D_{q}^{k} f(a) .
$$

Definition 3 [7] The fractional $q$-derivative of Caputo type of order $\alpha \geq 0$ on interval $[a, b]$ is defined by ${ }_{a}^{c} D_{q}^{0} f(t)=f(t)$ and

$$
\left({ }_{a}^{c} D_{q}^{\alpha} f\right)(t)=\left({ }_{a} I_{q}^{n-\alpha}{ }_{a} D_{q}^{n} f\right)(t), \alpha>0,
$$

where $n$ is the smallest integer greater than or equal to $\alpha$.

Lemma 4 [7] Let $\alpha>0$ and $n$ be the smallest integer great than or equal to $\alpha$. Then for $t \in[a, b]$ the following equality holds

$$
{ }_{a} I_{q a}^{\alpha c} D_{q}^{\alpha} f(t)=f(t)-\sum_{k=0}^{n-1} \frac{(t-a)^{k}}{\Gamma_{q}(k+1)}{ }_{a} D_{q}^{k} f(a) .
$$

\section{Main Results}

In this section, we will give the main results of this paper.

Let $P C(J, \mathbb{R})=\{x: J \rightarrow \mathbb{R}, x(t)$ is continuous everywhere except for some $t_{k}$ at which $x\left(t_{k}^{+}\right)$and $x\left(t_{k}^{-}\right)$exist, and $\left.x\left(t_{k}^{-}\right)=x\left(t_{k}\right), k=1,2, \cdots, m\right\}$. $P C(J, \mathbb{R})$ is a Banach space with the norm

$$
\|x\|=\sup \{|x(t)|: t \in J\} .
$$

First, for the sake of convenience, we introduce the following notations:

$$
\Lambda=\eta_{1}+\eta_{2}-\mu \sum_{i=0}^{m} \Omega_{\beta_{i}} \neq 0, \Omega_{\sigma_{i}}=\frac{t_{i}\left(t_{i+1}-t_{i}\right)_{q_{i}}^{\left(\sigma_{i}\right)}}{\Gamma_{q_{i}}\left(\sigma_{i}+1\right)},
$$

where $\sigma_{i} \in\left\{\alpha_{i}, \beta_{i}, \alpha_{i}+\beta_{i}\right\}, q_{i} \in(0,1), i=0,1,2, \cdots, m$.

To obtain our main results, we need the following lemma.

Lemma 5 Let $\mu \sum_{i=0}^{m} \Omega_{\beta_{i}} \neq \eta_{1}+\eta_{2}$ and $h(t) \in C(J, \mathbb{R})$. Then for any $t \in J_{k}$, 
the solution of the following problem

$$
\left\{\begin{array}{l}
{ }_{t_{k}}^{c} D_{q_{k}}^{\alpha_{k}} x(t)=h(t), t \in J_{k} \subseteq J=[0, T], t \neq t_{k}, \\
\Delta x\left(t_{k}\right)=x\left(t_{k}^{+}\right)-x\left(t_{k}\right)=\varphi_{k}\left(x\left(t_{k}\right)\right), k=1,2, \cdots, m, \\
\eta_{1} x(0)+\eta_{2} x(T)=\mu \sum_{k=0}^{m}{ }_{t_{k}} I_{q_{k}}^{\beta_{k}} x\left(t_{k+1}\right)
\end{array}\right.
$$

is given by

$$
\begin{aligned}
x(t)= & \frac{1}{\Lambda}\left\{\sum_{i=0}^{m}\left(\mu_{t_{i}} I_{q_{i}}^{\alpha_{i}+\beta_{i}} h\left(t_{i+1}\right)-\eta_{2 t_{i}} I_{q_{i}}^{\alpha_{i}} h\left(t_{i+1}\right)\right)\right. \\
& \left.+\sum_{i=1}^{m}\left[\mu\left(\sum_{j=1}^{i} \varphi_{j}\left(x\left(t_{j}\right)\right)+\sum_{j=0}^{i-1} t_{t_{j}} I_{q_{j}}^{\alpha_{j}} h\left(t_{j+1}\right)\right) \Omega_{\beta_{i}}-\eta_{2} \varphi_{i}\left(x\left(t_{i}\right)\right)\right]\right\} \\
& +\sum_{i=1}^{k} \varphi_{i}\left(x\left(t_{i}\right)\right)+\sum_{i=0}^{k-1}{ }_{t_{i}} I_{q_{i}}^{\alpha_{i}} h\left(t_{i+1}\right)+{ }_{t_{k}} I_{q_{k}}^{\alpha_{k}} h(t) .
\end{aligned}
$$

Proof. Applying the operator ${ }_{t_{0}} I_{q_{0}}^{\alpha_{0}}$ on both sides of the first equation of (2) for $t \in J_{0}$ and using Lemma 4, we have

$$
x(t)=x\left(t_{0}\right)+{ }_{t_{0}} I_{q_{0}}^{\alpha_{0}} h(t) .
$$

Then we get for $t=t_{1}$ that

$$
x\left(t_{1}\right)=x\left(t_{0}\right)+{ }_{t_{0}} I_{q_{0}}^{\alpha_{0}} h\left(t_{1}\right) .
$$

For $t \in J_{1}$, again taking the ${ }_{t_{1}} I_{q_{1}}^{\alpha_{1}}$ to (4) and using the above process, we get

$$
x(t)=x\left(t_{1}^{+}\right)+{ }_{t_{1}} I_{q_{1}}^{\alpha_{1}} h(t) .
$$

Applying the impulsive condition $x\left(t_{1}^{+}\right)=x\left(t_{1}\right)+\varphi_{1}\left(x\left(t_{1}\right)\right)$, we get

$$
x(t)=x\left(t_{0}\right)+\varphi_{1}\left(x\left(t_{1}\right)\right)+{ }_{t_{0}} I_{q_{0}}^{\alpha_{0}} h\left(t_{1}\right)+{ }_{t_{1}} I_{q_{1}}^{\alpha_{1}} h(t) .
$$

By the same way, for $t \in J_{2}$, we have

$$
x(t)=x\left(t_{0}\right)+\varphi_{1}\left(x\left(t_{1}\right)\right)+\varphi_{2}\left(x\left(t_{2}\right)\right)+{ }_{t_{0}} I_{q_{0}}^{\alpha_{0}} h\left(t_{1}\right)+{ }_{t_{1}} I_{q_{1}}^{\alpha_{1}} h\left(t_{2}\right)+{ }_{t_{2}} I_{q_{2}}^{\alpha_{2}} h(t) .
$$

Repeating the above process for $t \in J_{k} \subseteq J, k=0,1,2, \cdots, m$, we get

$$
x(t)=x\left(t_{0}\right)+\sum_{i=1}^{k} \varphi_{i}\left(x\left(t_{i}\right)\right)+\sum_{i=0}^{k-1}{ }_{t_{i}} I_{q_{i}}^{\alpha_{i}} h\left(t_{i+1}\right)+{ }_{t_{k}} I_{q_{k}}^{\alpha_{k}} h(t) .
$$

From (5), we find that

$$
x(T)=x\left(t_{0}\right)+\sum_{i=1}^{k} \varphi_{i}\left(x\left(t_{i}\right)\right)+\sum_{i=0}^{k-1}{ }_{t_{i}} I_{q_{i}}^{\alpha_{i}} h\left(t_{i+1}\right)+{ }_{t_{k}} I_{q_{k}}^{\alpha_{k}} h(T) .
$$

From the boundary condition of (2), we get

$$
\begin{aligned}
x\left(t_{0}\right)= & \frac{1}{\Lambda}\left\{\sum_{i=0}^{m}\left(\mu_{t_{i}} I_{q_{i}}^{\alpha_{i}+\beta_{i}} h\left(t_{i+1}\right)-\eta_{2 t_{i}} I_{q_{i}}^{\alpha_{i}} h\left(t_{i+1}\right)\right)\right. \\
& \left.+\sum_{i=1}^{m}\left[\mu\left(\sum_{j=1}^{i} \varphi_{j}\left(x\left(t_{j}\right)\right)+\sum_{j=0}^{i-1}{ }_{t_{j}} I_{q_{j}}^{\alpha_{j}} h\left(t_{j+1}\right)\right) \Omega_{\beta_{i}}-\eta_{2} \varphi_{i}\left(x\left(t_{i}\right)\right)\right]\right\} .
\end{aligned}
$$

Substituting (6) to (5), we obtain the solution (3). This completes the proof. 
We define an operator $\mathcal{G}: P C(J, \mathbb{R}) \rightarrow P C(J, \mathbb{R})$ as follows:

$$
\begin{aligned}
\mathcal{G} x(t)= & \frac{1}{\Lambda}\left\{\sum_{i=0}^{m}\left(\mu_{t_{i}} I_{q_{i}}^{\alpha_{i}+\beta_{i}} f(s, x)\left(t_{i+1}\right)-\eta_{2 t_{i}} I_{q_{i}}^{\alpha_{i}} f(s, x)\left(t_{i+1}\right)\right)\right. \\
& \left.+\sum_{i=1}^{m}\left[\mu\left(\sum_{j=1}^{i} \varphi_{j}\left(x\left(t_{j}\right)\right)+\sum_{j=0}^{i-1}{ }_{t_{j}} I_{q_{j}}^{\alpha_{j}} f(s, x)\left(t_{j+1}\right)\right) \Omega_{\beta_{i}}-\eta_{2} \varphi_{i}\left(x\left(t_{i}\right)\right)\right]\right\} \\
& +\sum_{i=1}^{k} \varphi_{i}\left(x\left(t_{i}\right)\right)+\sum_{i=0}^{k-1} t_{t_{i}} I_{q_{i}}^{\alpha_{i}} f(s, x)\left(t_{i+1}\right)+{ }_{t_{k}} I_{q_{k}}^{\alpha_{k}} f(s, x)(t) .
\end{aligned}
$$

Then, the existence of solutions of system (1) is equivalent to the problem of fixed point of operator $\mathcal{G}$ in (7).

Theorem 1 Let $f: J \times \mathbb{R}_{m} \rightarrow \mathbb{R}$ and $\varphi_{k}: \mathbb{R} \rightarrow \mathbb{R}, k=1,2, \cdots, m$ be continuous functions. Assume that $\mu \sum_{i=0}^{m} \Omega_{\beta_{i}} \neq \eta_{1}+\eta_{2}$ and the following conditions are satisfied:

$\left(\mathrm{H}_{1}\right)$ There exists a positive constant $L$ such that $\left|\varphi_{k}(x)-\varphi_{k}(y)\right| \leq L|x-y|$ for each $x, y \in \mathbb{R}$ and $k=1,2, \cdots, m$.

$\left(\mathrm{H}_{2}\right)$ There exists a function $M(t) \in C\left(J, \mathbb{R}^{+}\right)$such that

$$
|f(t, x)-f(t, y)| \leq M(t)|x-y|, \forall t \in J, x, y \in \mathbb{R} .
$$

$\left(\mathrm{H}_{3}\right) \quad \Delta<1$.

Then problem (1) has a unique solution on $J$, where $M=\sup _{t \in J}|M(t)|$ and

$$
\begin{aligned}
\Delta= & \frac{1}{\Lambda} \sum_{i=1}^{m}\left(\mu M \Omega_{\alpha_{i}+\beta_{i}}+\left(\eta_{2}+M\right) \Omega_{\alpha_{i}}+\mu M \sum_{j=0}^{i-1} \Omega_{\alpha_{j}} \Omega_{\beta_{i}}+\mu L i \Omega_{\beta_{i}}\right) \\
& +\frac{1}{\Lambda}\left(\mu \Omega_{\alpha_{0}+\beta_{0}}+\eta_{2} \Omega_{\alpha_{0}}\right)+m L\left(\frac{1}{\Lambda} \eta_{2}+1\right) .
\end{aligned}
$$

Proof. The conclusion will follow once we have shown that the operator $\mathcal{G}$ defined (7) is a construction with respect to a suitable norm on $P C(J, \mathbb{R})$.

For any functions $x, y \in P C(J, \mathbb{R})$, we have

$$
\begin{aligned}
& |(\mathcal{G} x)(t)-(\mathcal{G} y)(t)| \\
& \leq \frac{1}{\Lambda}\left\{\sum_{i=0}^{m}\left(\mu_{t_{i}} I_{q_{i}}^{\alpha_{i}+\beta_{i}}|f(s, x)-f(s, y)|\left(t_{i+1}\right)+\eta_{2} t_{i} I_{q_{i}}^{\alpha_{i}}|f(s, x)-f(s, y)|\left(t_{i+1}\right)\right)\right. \\
& \quad+\sum_{i=1}^{m}\left[\mu\left(\sum_{j=1}^{i}\left|\varphi_{j}\left(x\left(t_{j}\right)\right)-\varphi_{j}\left(y\left(t_{j}\right)\right)\right|+\sum_{j=0}^{i-1} t_{t_{j}} I_{q_{j}}^{\alpha_{j}}|f(s, x)-f(s, y)|\left(t_{j+1}\right)\right) \Omega_{\beta_{i}}\right. \\
& \left.\left.+\eta_{2}\left|\varphi_{i}\left(x\left(t_{i}\right)\right)-\varphi_{i}\left(y\left(t_{i}\right)\right)\right|\right]\right\}+\sum_{i=1}^{m}\left|\varphi_{i}\left(x\left(t_{i}\right)\right)-\varphi_{i}\left(y\left(t_{i}\right)\right)\right| \\
& +\sum_{i=0}^{m-1}{ }_{t_{i}} I_{q_{i}}^{\alpha_{i}}|f(s, x)-f(s, y)|\left(t_{i+1}\right)+{ }_{t_{m}} I_{q_{m}}^{\alpha_{m}}|f(s, x)-f(s, y)|(t) .
\end{aligned}
$$

By conditions $\left(\mathrm{H}_{1}\right)$ and $\left(\mathrm{H}_{2}\right)$, we get

$$
\begin{aligned}
& |(\mathcal{G} x)(t)-(\mathcal{G} y)(t)| \\
& \leq \frac{1}{\Lambda}\left\{\sum_{i=0}^{m}\left(\mu_{t_{i}} I_{q_{i}}^{\alpha_{i}+\beta_{i}}(M\|x-y\|)\left(t_{i+1}\right)+\eta_{2 t_{i}} I_{q_{i}}^{\alpha_{i}}(M\|x-y\|)\left(t_{i+1}\right)\right)\right. \\
& \left.\quad+\sum_{i=1}^{m}\left[\mu\left(\sum_{j=1}^{i} L\|x-y\|+\sum_{j=0}^{i-1} t_{j} I_{q_{j}}^{\alpha_{j}}(M\|x-y\|)\right) \Omega_{\beta_{i}}+\eta_{2} L\|x-y\|\right]\right\}
\end{aligned}
$$




$$
\begin{aligned}
& +\sum_{i=1}^{m} L\|x-y\|+\sum_{i=0}^{m-1}{ }_{t_{i}} I_{q_{i}}^{\alpha_{i}}(M\|x-y\|)\left(t_{i+1}\right)+{ }_{t_{m}} I_{q_{m}}^{\alpha_{m}}(M\|x-y\|)\left(t_{m+1}\right) \\
\leq & \left\{\frac{1}{\Lambda} \sum_{i=1}^{m}\left(\mu M \Omega_{\alpha_{i}+\beta_{i}}+\eta_{2} \Omega_{\alpha_{i}}+\mu L i \Omega_{\beta_{i}}+\mu M \sum_{j=0}^{i-1} \Omega_{\alpha_{j}} \Omega_{\beta_{i}}+M \Omega_{\alpha_{i}}\right)\right. \\
& \left.+\frac{1}{\Lambda}\left(\mu \Omega_{\alpha_{0}+\beta_{0}}+\eta_{2} \Omega_{\alpha_{0}}\right)+m L\left(\frac{1}{\Lambda} \eta_{2}+1\right)\right\}\|x-y\|,
\end{aligned}
$$

which implies that

$$
\|\mathcal{G} x-\mathcal{G} y\| \leq \Delta\|x-y\|
$$

Thus the operator $\mathcal{G}$ is a contraction in view of the condition $\left(\mathrm{H}_{3}\right)$. By Banach's contraction mapping principle, the problem (1) has a unique solution on $J$. This completes the proof.

In the following, we study the Hyers-Ulam stability of impulsive fractional $q$-difference Equation (1). Let $\varepsilon>0, \epsilon>0$ and $\delta:[0, T] \rightarrow \mathbb{R}$ be a continuous function. Consider the inequalities:

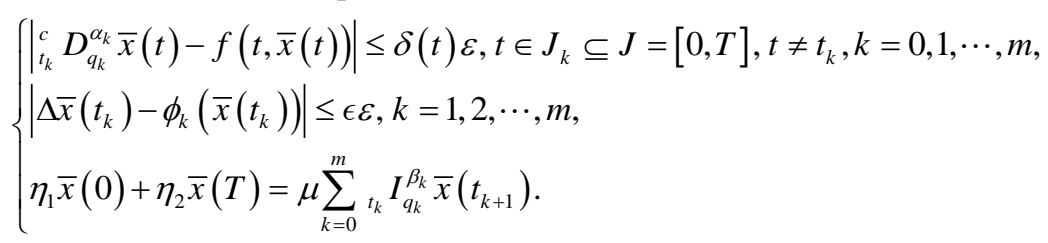

Now, we give out the definition of Hyers-Ulam stability of system (1).

Definition 4 System (1) is Hyers-Ulam stable with respect to system (8), if there exists $A_{f}>0$ such that

$$
|\bar{x}-\tilde{x}| \leq A_{f} \varepsilon
$$

for all $t \in J$, where $\bar{X}$ is the solution of (8), and $\tilde{X}$ of the solution for system (1).

Theorem 2 Assume $f: J \times \mathbb{R} \rightarrow \mathbb{R}$ satisfy assumption $\left(\mathrm{H}_{2}\right)$, $\varphi_{i}: \mathbb{R} \rightarrow \mathbb{R}, i=1,2, \cdots, m$ are continuous functions and satisfy assumption $\left(\mathrm{H}_{1}\right)$ and the condition $\left(\mathrm{H}_{3}\right)$ holds, $\sup \delta(t) \leq 1$. Then the system (1) is Hyers-Ulam stable with respect to system $(8){ }^{t \in J}$

Proof. Let ${ }_{t_{k}}^{c} D_{q_{k}}^{\alpha_{k}} \bar{x}(t)=f(t, \bar{x}(t))+g(t), k=0,1, \cdots, m$ and $\Delta \bar{x}\left(t_{k}\right)=\varphi_{k}\left(\bar{x}\left(t_{k}\right)\right)+g_{k}, k=1,2, \cdots, m$. Consider the system

$$
\left\{\begin{array}{l}
{ }_{t_{k}}^{c} D_{q_{k}}^{\alpha_{k}} \bar{x}(t)=f(t, \bar{x}(t))+g(t), t \in J_{k} \subseteq J=[0, T], t \neq t_{k}, \\
\Delta \bar{x}\left(t_{k}\right)=\varphi_{k}\left(\bar{x}\left(t_{k}\right)\right)+g_{k}, k=1,2, \cdots, m . \\
\eta_{1} \bar{x}(0)+\eta_{2} \bar{x}(T)=\mu \sum_{k=0}^{m} I_{t_{k}} I_{q_{k}}^{\beta_{k}} \bar{x}\left(t_{k+1}\right) .
\end{array}\right.
$$

Similarly to the system in Theorem 1, system (9) is equivalent to the following integral equation in Lemma 5.

$$
\begin{aligned}
\bar{x}(t)= & \frac{1}{\Lambda}\left\{\sum_{i=0}^{m}\left(\mu_{t_{i}} I_{q_{i}}^{\alpha_{i}+\beta_{i}}(f(s, \bar{x})+g(s))\left(t_{i+1}\right)-\eta_{2 t_{i}} I_{q_{i}}^{\alpha_{i}}(f(s, \bar{x})+g(s))\left(t_{i+1}\right)\right)\right. \\
& +\sum_{i=1}^{m}\left[\mu\left(\sum_{j=1}^{i}\left(\varphi_{j}\left(\bar{x}\left(t_{j}\right)\right)+g_{j}\right)+\sum_{j=0}^{i-1} t_{t_{j}} I_{q_{j}}^{\alpha_{j}}(f(s, \bar{x})+g(s))\left(t_{j+1}\right)\right) \Omega_{\beta_{i}}\right.
\end{aligned}
$$




$$
\begin{aligned}
& \left.\left.-\eta_{2}\left(\varphi_{i}\left(\bar{x}\left(t_{i}\right)\right)+g_{i}\right)\right]\right\}+\sum_{i=1}^{k}\left(\varphi_{i}\left(\bar{x}\left(t_{i}\right)\right)+g_{i}\right) \\
& +\sum_{i=0}^{k-1} I_{t_{i}} I_{q_{i}}^{\alpha_{i}}(f(s, \bar{x})+g(s))\left(t_{i+1}\right)+{ }_{t_{k}} I_{q_{k}}^{\alpha_{k}}(f(t, \bar{x})+g(t))
\end{aligned}
$$

Now, we define the operator $\tilde{\mathcal{G}}$ as following

$$
\begin{aligned}
\tilde{\mathcal{G}} x(t)= & \frac{1}{\Lambda}\left\{\sum_{i=0}^{m}\left(\mu_{t_{i}} I_{q_{i}}^{\alpha_{i}+\beta_{i}} f(s, x)\left(t_{i+1}\right)-\eta_{2 t_{i}} I_{q_{i}}^{\alpha_{i}} f(s, x)\left(t_{i+1}\right)\right)\right. \\
& \left.+\sum_{i=1}^{m}\left[\mu\left(\sum_{j=1}^{i} \varphi_{j}\left(x\left(t_{j}\right)\right)+\sum_{j=0}^{i-1}{ }_{t_{j}} I_{q_{j}}^{\alpha_{j}} f(s, x)\left(t_{j+1}\right)\right) \Omega_{\beta_{i}}-\eta_{2} \varphi_{i}\left(x\left(t_{i}\right)\right)\right]\right\} \\
& +\sum_{i=1}^{k} \varphi_{i}\left(x\left(t_{i}\right)\right)+\sum_{i=0}^{k-1}{ }_{t_{i}} I_{q_{i}}^{\alpha_{i}} f(s, x)\left(t_{i+1}\right)+{ }_{t_{k}} I_{q_{k}}^{\alpha_{k}} f(s, x)(t)+G(t) \\
= & \mathcal{G} x+G(t) .
\end{aligned}
$$

where

$$
\begin{aligned}
G(t)= & \frac{1}{\Lambda}\left\{\sum_{i=0}^{m}\left(\mu_{t_{i}} I_{q_{i}}^{\alpha_{i}+\beta_{i}} g\left(t_{i+1}\right)-\eta_{2} I_{t_{i}} I_{q_{i}}^{\alpha_{i}} g\left(t_{i+1}\right)\right)\right. \\
& \left.+\sum_{i=1}^{m}\left[\mu\left(\sum_{j=1}^{i} g_{j}+\sum_{j=0}^{i-1}{ }_{t_{j}} I_{q_{j}}^{\alpha_{j}} g\left(t_{j+1}\right)\right) \Omega_{\beta_{i}}-\eta_{2} g_{i}\right]\right\} \\
& +\sum_{i=1}^{k} g_{i}+\sum_{i=0}^{k-1}{ }_{t_{i}} I_{q_{i}}^{\alpha_{i}} g\left(t_{i+1}\right)+{ }_{t_{k}} I_{q_{k}}^{\alpha_{k}} g(t) .
\end{aligned}
$$

Note that

$$
\|\tilde{\mathcal{G}} x-\tilde{\mathcal{G}} y\|=\|\mathcal{G} x-\mathcal{G} y\| .
$$

Then the existence of a solution of (1) implies the existence of a solution to (9), it follows from Theorem 1 that $\tilde{\mathcal{G}}$ is a contraction. Thus there is a unique fixed point $\bar{x}$ of $\tilde{\mathcal{G}}$, and respectively $\tilde{X}$ of $\mathcal{G}$.

Since $t \in[0, T]$ and $\sup _{t \in J} \delta(t) \leq 1$, we obtain

$$
\begin{aligned}
\|G\|= & \max _{t \in J}|G(t)| \\
= & \max _{t \in J} \mid \frac{1}{\Lambda}\left\{\sum_{i=0}^{m}\left(\mu_{t_{i}} I_{q_{i}}^{\alpha_{i}+\beta_{i}} g\left(t_{i+1}\right)-\eta_{2 t_{i}} I_{q_{i}}^{\alpha_{i}} g\left(t_{i+1}\right)\right)\right. \\
& \left.+\sum_{i=1}^{m}\left[\mu\left(\sum_{j=1}^{i} g_{j}+\sum_{j=0}^{i-1}{ }_{t_{j}} I_{q_{j}}^{\alpha_{j}} g\left(t_{j+1}\right)\right) \Omega_{\beta_{i}}-\eta_{2} g_{i}\right]\right\} \\
& +\sum_{i=1}^{k} g_{i}+\sum_{i=0}^{k-1} t_{i} I_{q_{i}}^{\alpha_{i}} g\left(t_{i+1}\right)+{ }_{t_{k}} I_{q_{k}}^{\alpha_{k}} g(t) \mid \\
\leq & \max _{t \in J} \mid \frac{1}{\Lambda}\left\{\sum_{i=0}^{m}\left(\mu_{t_{i}} I_{q_{i}}^{\alpha_{i}+\beta_{i}} g\left(t_{i+1}\right)-\eta_{2} I_{t_{i}} I_{q_{i}}^{\alpha_{i}} g\left(t_{i+1}\right)\right)\right. \\
& \left.+\sum_{i=1}^{m}\left[\mu\left(\sum_{j=1}^{i} g_{j}+\sum_{j=0}^{i-1} t_{t_{j}} I_{q_{j}}^{\alpha_{j}} g\left(t_{j+1}\right)\right) \Omega_{\beta_{i}}-\eta_{2} g_{i}\right]\right\}
\end{aligned}
$$




$$
\begin{aligned}
& +\sum_{i=1}^{m} g_{i}+\sum_{i=0}^{m-1} t_{t_{i}} I_{q_{i}}^{\alpha_{i}} g\left(t_{i+1}\right)+{ }_{t_{m}} I q_{m} \alpha_{m} g(t) \mid \\
\leq & \left\{\frac{1}{\Lambda} \sum_{i=1}^{m}\left(\mu \Omega_{\alpha_{i}+\beta_{i}}+\eta_{2} \Omega_{\alpha_{i}}+\mu \epsilon i \Omega_{\beta_{i}}+\mu \sum_{j=0}^{i-1} \Omega_{\alpha_{j}} \Omega_{\beta_{i}}+\Omega_{\alpha_{i}}\right)\right. \\
& \left.+\frac{1}{\Lambda}\left(\mu \Omega_{\alpha_{0}+\beta_{0}}+\eta_{2} \Omega_{\alpha_{0}}\right)+m \epsilon\left(\frac{1}{\Lambda} \eta_{2}+1\right)\right\} \varepsilon
\end{aligned}
$$

Then, we get

$$
\begin{aligned}
\|\bar{x}-\tilde{x}\|= & \|\tilde{\mathcal{G}} \bar{x}-\mathcal{G} \tilde{x}\|=\|\mathcal{G} \bar{x}-\mathcal{G} \tilde{x}+G(t)\| \leq\|\mathcal{G} \bar{x}-\mathcal{G} \tilde{x}\|+\|G\| \\
\leq & \Delta\|\bar{x}-\tilde{x}\|+\left\{\frac{1}{\Lambda} \sum_{i=0}^{m}\left(\mu \Omega_{\alpha_{i}+\beta_{i}}+\eta_{2} \Omega_{\alpha_{i}}+\mu \epsilon i \Omega_{\beta_{i}}+\mu \sum_{j=0}^{i-1} \Omega_{\alpha_{j}} \Omega_{\beta_{i}}+\Omega_{\alpha_{i}}\right)\right. \\
& \left.+\frac{1}{\Lambda}\left(\mu \Omega_{\alpha_{0}+\beta_{0}}+\eta_{2} \Omega_{\alpha_{0}}\right)+m \epsilon\left(\frac{1}{\Lambda} \eta_{2}+1\right)\right\} \varepsilon .
\end{aligned}
$$

By condition $\left(\mathrm{H}_{3}\right)$, we have

$$
\begin{aligned}
\|\bar{x}-\tilde{x}\| \leq & (1-\Delta)^{-1}\left\{\frac{1}{\Lambda} \sum_{i=0}^{m}\left(\mu \Omega_{\alpha_{i}+\beta_{i}}+\eta_{2} \Omega_{\alpha_{i}}+\mu \epsilon i \Omega_{\beta_{i}}+\mu \sum_{j=0}^{i-1} \Omega_{\alpha_{j}} \Omega_{\beta_{i}}+\Omega_{\alpha_{i}}\right)\right. \\
& \left.+m \epsilon\left(\frac{1}{\Lambda} \eta_{2}+1\right)\right\} \varepsilon .
\end{aligned}
$$

Let

$$
A_{f}=(1-\Delta)^{-1}\left\{\frac{1}{\Lambda} \sum_{i=0}^{m}\left(\mu \Omega_{\alpha_{i}+\beta_{i}}+\eta_{2} \Omega_{\alpha_{i}}+\mu \epsilon i \Omega_{\beta_{i}}+\mu \sum_{j=0}^{i-1} \Omega_{\alpha_{j}} \Omega_{\beta_{i}}+\Omega_{\alpha_{i}}\right)\right. \text {, then }
$$

$$
\left.+m \epsilon\left(\frac{1}{\Lambda} \eta_{2}+1\right)\right\}
$$

$\|\bar{x}-\tilde{x}\| \leq A_{f} \varepsilon$.

This completes the proof.

Remark 1 Note that (1) has a very general form, as special instances results from (1), when, $\eta_{1}=\eta_{2}=1, \mu=0$, (1) reduces to the antiperiodic boundary value problem of the impulsive fractional $q$-difference equation:

$$
\left\{\begin{array}{l}
{ }_{t_{k}} D_{q_{k}}^{\alpha_{k}} x(t)=f(t, x(t)), t \in J_{k} \subseteq J=[0, T], t \neq t_{k}, \\
\Delta x\left(t_{k}\right)=x\left(t_{k}^{+}\right)-x\left(t_{k}\right)=\varphi_{k}\left(x\left(t_{k}\right)\right), k=1,2, \cdots, m, \\
x(0)+x(T)=0
\end{array}\right.
$$

\section{Example}

Consider the following boundary value problem:

$$
\left\{\begin{array}{l}
c_{t_{k}} D_{\frac{3 k+1}{4 k+3}}^{\frac{k+1}{3 k+2}} x(t)=\frac{\sin ^{2} t}{t^{2}+50} \frac{2|x(t)|}{1+|x(t)|}+\frac{3 t}{4}, t \in\left[0, \frac{3}{2}\right] \backslash\left\{t_{1}, t_{2}\right\}, \\
\Delta x\left(t_{k}\right)=\frac{1}{200 k} \frac{x^{2}\left(t_{k}\right)+2\left|x\left(t_{k}\right)\right|}{1+\left|x\left(t_{k}\right)\right|}+\frac{k}{5}, t_{k}=\frac{k}{2}, k=1,2, \\
\frac{8}{3} x(0)+\frac{1}{6} x\left(\frac{3}{2}\right)=\frac{1}{2} \sum_{k=0}^{2} t_{t_{k}} \frac{\frac{k+1}{k^{2}+2}}{4 k+3} x\left(t_{k+1}\right) .
\end{array}\right.
$$


Corresponding to boundary value problem (1), one see that $\alpha_{k}=\frac{k+1}{3 k+2}$, $\beta_{k}=\frac{k+1}{k^{2}+2}, \quad q_{k}=\frac{3 k+1}{4 k+3}, t_{k}=\frac{k}{2}, \quad f(t, x)=\frac{\sin ^{2} t}{t^{2}+50} \frac{2|x(t)|}{1+|x(t)|}+\frac{3}{4}$, $\varphi_{k}\left(x\left(t_{k}\right)\right)=\frac{1}{200 k} \frac{x^{2}\left(t_{k}\right)+2\left|x\left(t_{k}\right)\right|}{1+\left|x\left(t_{k}\right)\right|}$. Through a simple calculation, we get

$$
\begin{gathered}
|f(t, x)-f(t, y)| \leq \frac{\sin ^{2} t}{t^{2}+25}|x-y|, M(t)=\frac{\sin ^{2} t}{t^{2}+25} \leq \frac{1}{25}=M, \\
\left|\varphi_{k}(x)-\varphi_{k}(y)\right| \leq \frac{1}{200 k}|x-y| \leq \frac{1}{200}|x-y|, L=\frac{1}{200}, \\
\Lambda \doteq 1.7875>0, \Delta \doteq 0.4873<1 .
\end{gathered}
$$

From Theorem 1, the problem (16) has a unique solution $x$ on $\left[0, \frac{3}{2}\right]$. Furthermore, the solution $x$ is Hyers-Ulam stable with respect to the following system

$$
\begin{aligned}
& ||\left|c \frac{c}{t_{k}} \frac{\frac{k+1}{3 k+2}}{3 k+1} x(t)-\frac{\sin ^{2} t}{t^{2}+50} \frac{2|x(t)|}{1+|x(t)|}-\frac{3 t}{4}\right| \leq \delta(t) \varepsilon, t \in\left[0, \frac{3}{2}\right] \backslash\left\{t_{1}, t_{2}\right\}, \\
& \left\{\left|\Delta x\left(t_{k}\right)-\frac{1}{200 k} \frac{x^{2}\left(t_{k}\right)+2\left|x\left(t_{k}\right)\right|}{1+\left|x\left(t_{k}\right)\right|}-\frac{k}{5}\right| \leq \epsilon \varepsilon, t_{k}=\frac{k}{2}, k=1,2,\right. \\
& \frac{8}{3} x(0)+\frac{1}{6} x\left(\frac{3}{2}\right)=\frac{1}{2} \sum_{k=0}^{2} t_{k} I_{\frac{k k+1}{4 k+3}}^{\frac{k+1}{k^{2}+2}} x\left(t_{k+1}\right),
\end{aligned}
$$

where $\epsilon>0, \varepsilon>0, \sup _{t \in\left[0, \frac{3}{2}\right]} \delta(t)<1$.

\section{Conclusion}

In this paper, we study the existence and Hyers-Ulam stability of solutions for impulsive fractional $q$-difference equation. We obtain some results as following: 1) Using the $q$-shifting operator, the results of existence of solutions for impulsive fractional $q$-difference equation with $q$-integral boundary conditions are obtained. 2) The Hyers-Ulam stability of the nonlinear impulsive fractional $q$-difference equations was obtained.

\section{Funding}

This research was supported by Science and Technology Foundation of Guizhou Province (Grant No. [2016] 7075), by the Project for Young Talents Growth of Guizhou Provincial Department of Education under (Grant No. Ky [2017] 133), and by the project of Guizhou Minzu University under (Grant No.16yjrcxm002).

\section{Conflicts of Interest}

The authors declare no conflicts of interest regarding the publication of this paper. 


\section{References}

[1] Jackson, F.H. (1910) On $q$-Definite Integrals. The Quarterly Journal of Pure and Applied Mathematics, 41, 193-203.

[2] Kac, V. and Cheung, P. (2002) Quantum Calculus. Springer, New York. https://doi.org/10.1007/978-1-4613-0071-7

[3] Al-Salam, W.A. (1966) Some Fractional $q$-Integral and $q$-Derivatives. Proceedings of the Edinburgh Mathematical Society, 15, 135-140. https://doi.org/10.1017/S0013091500011469

[4] Agarwal, R.P. (1969) Certain Fractional $q$-Integrals and $q$-Derivatives. Mathematical Proceedings of the Cambridge Philosophical Society, 66, 365-370. https://doi.org/10.1017/S0305004100045060

[5] Tariboom, T., Ntouvas, S.K. and Agarwal, R. (2015) New Concepts of Fractional Quantum Calculus and Applications to Impulsive Fractional $q$-Difference Equation. Advances in Difference Equations, 2015, Article No. 18. https://doi.org/10.1186/s13662-014-0348-8

[6] Tariboom, T. and Ntouvas, S.K. (2013) Quantum Calculus on Finite Intervals and Applications to Impulsive Difference Equations. Advances in Difference Equations, 2013, Article No. 282. https://doi.org/10.1186/1687-1847-2013-282

[7] Ahmad, B., Ntouyas, S.K., Tariboon, J., Alsaedi, A. and Alsulami, H.H. (2016) Impulsive Fractional $q$-Integro-Difference Equations with Separated Boundary Conditions. Applied Mathematics and Computation, 281, 199-213. https://doi.org/10.1016/j.amc.2016.01.051

[8] Agarwal, R.P., Wang, G., Ahmad, B., Zhang, L., Hobiny, A. and Monaquel, S. (2015) On Existence of Solutions for Nonlinear $q$-Difference Equations with Nonlocal $q$-Integral Boundary Conditions. Mathematical Modelling and Analysis, 20, 604-618. https://doi.org/10.3846/13926292.2015.1088483

[9] Li, X.H., Han, Z.L., Sun, S.R. and Sun, L.Y. (2016) Eigenvalue Problems of Fractional $q$-Difference Equations with Generalized $p$-Laplacian. Applied Mathematics Letters, 57, 46-53. https://doi.org/10.1016/j.aml.2016.01.003

[10] Ferreira, R. (2011) Positive Solutions for a Class of Boundary Value Problems with Fractional $q$-Differences. Computers \& Mathematics with Applications, 61, 367-373. https://doi.org/10.1016/j.camwa.2010.11.012

[11] Ge, Q. and Hou, C.M. (2015) Positive Solution for a Class of $p$-Laplacian Fractional $q$-Difference Equations Involving the Integral Boundary Condition. Mathematica Aeterna, 5, 927-944.

[12] Balkani, N., Rezapour, S. and Haghi, R.H. (2019) Approximate Solutions for a Fractional $q$-Integro-Difference Equation. Journal of Mathematical Extension, 13, 201-214.

[13] Samei, M.E. and Khalilzadeh Ranjbar, G. (2019) Some Theorems of Existence of Solutions for Fractional Hybrid $q$-Difference Inclusion. Journal of Advanced Mathematical Studies, 12, 63-76. https://doi.org/10.1186/s13662-019-2090-8

[14] Kalvandi, V. and Samei, M.E. (2019) New Stability Results for a Sum-Type Fractional $q$-Integro-Differential Equation. Journal of Advanced Mathematical Studies, 12, 201-209.

[15] Liang, S.H. and Samei, M.E. (2020) New Approach to Solutions of a Class of Singular Fractional $q$-Differential Problem via Quantum Calculus. Advances in Difference Equations, 2020, Article No. 14. https://doi.org/10.1186/s13662-019-2489-2

[16] Zhai, C.B. and Ren, J. (2018) The Unique Solution for a Fractional $q$-Difference Equation with Three-Point Boundary Conditions. Indagationes Mathematicae, 29, 948-961. https://doi.org/10.1016/j.indag.2018.02.002 
[17] Fahd, J., Thabet, A. and Dumitru, B. (2013) Stability of $q$-Fractional Non-Autonomous Systems. Nonlinear Analysis, 14, 780-784.

https://doi.org/10.1016/j.nonrwa.2012.08.001 